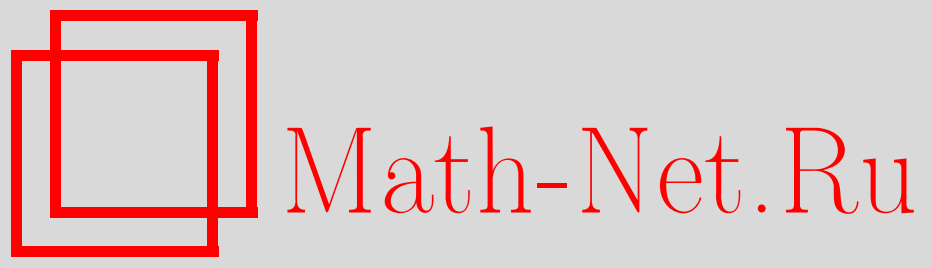

К. Кухиеро, И. Кляйн, Й. Тайхманн, Новый взгляд на фундаментальную теорему теории арбитража для больших финансовых рынков, Теория вероятн. и ее примен., 2015, том 60, выпуск 4, 660-685

DOI: https://doi.org/10.4213/tvp5030

Использование Общероссийского математического портала Math-Net.Ru подразумевает, что вы прочитали и согласны с пользовательским соглашением http://www.mathnet.ru/rus/agreement

Параметры загрузки:

IP: 3.91 .87 .62

26 апреля 2023 г., 09:27:57

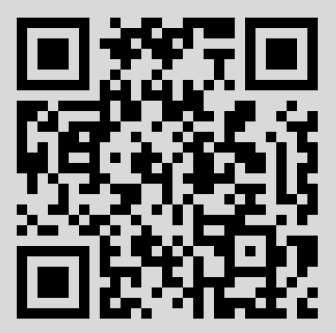




\title{
НОВЫЙ ВЗГЛЯД НА ФУНДАМЕНТАЛЬНУЮ ТЕОРЕМУ ТЕОРИИ АРБИТРАЖА ДЛЯ БОЛЬШИХ ФИНАНСОВЫХ РЫНКОВ
}

\author{
(Перевод Лещенко С.С.)
}

В контексте больших финансовых рынков мы формулируем понятие отсутствия асимптотического бесплатного ленча с исчезаюшим риском (NAFLVR), в соответствии с которым мы можем доказать версию фундаментальной теоремы теории арбитража (FTAP) в рынках с (даже несчетным) бесконечным числом активов, как, например, в случае рынков облигаций. Мы работаем в общей модели допустимых процессов капитала портфелей, предложенной Ю. Кабановым [8], в рамках существенно ослабленного свойства конкатенации и адаптируем вариант доказательства FTAP, полученный в [1] для классической ситуации малого рынка, к большим финансовым рынкам. В случае счетного числа активов наша ситуация включает модель большого финансового рынка, рассмотренную М. Де Донно и др. в [2], и их абстрактную теорию интегрирования.

Понятие (NAFLVR) оказывается экономически значимым условием «отсутствия арбитража» (в частности, не связанным со *слабыми замыканиями), и (NAFLVR) эквивалентно существованию разделяющей меры. Более того, мы покажем с помощью контрпримера, что существование эквивалентной разделяющей меры не приводит к эквивалентной $\sigma$-мартингальной мере даже в ситуации счетного большого финансового рынка.

Ключевые слова и фразы: фундаментальная теорема теории арбитража, большие финансовые рынки, топология Эмери, условие (NFLVR), свойство (P-UT).

1. Введение. В финансовой математике классическая модель рынка состоит из $\mathbb{R}^{d}$-значного семимартингала на некотором фильтрованном вероятностном пространстве, который описывает процесс дисконтированных цен $d$ финансовых активов. В терминологии настоящей статьи, такая модель называется малыл финансовым рынком, в отличие от большого финансового рынка, который соответствует последовательности малых рынков. Это понятие было введено Ю. Кабановым

*Vienna University, Oskar-Morgenstern-Platz 1, A-1090 Vienna; ETH Zürich, Rämistrasse 101, CH-8092, Zürich; e-mail: christa.cuchiero@univie.ac.at; irene.klein@ univie.ac.at; jteichma@math.ethz.ch

1) Авторы выражают признательность ЕTH-foundation за поддержку. 
и Д. Крамковым [9], которые рассмотрели последовательность, индексированную $n, \mathbb{R}^{d(n)}$-значных семимартингалов на, возможно, различных вероятностных пространствах. Для такой модели большого финансового рынка несколько понятий и характеризаций асимптотического арбитража были разработаны в [9], [14], [13], [10]. В частности, версия фундаментальной теоремы теории арбитража (FTAP) была доказана в [13] путем адаптации понятия отсутствия бесплатного ленча (NFL) Д. Крепса [16] к ситуации больших финансовых рынков. В упрощенной ситуации одного фиксированного вероятностного пространства это соответствует теореме Крепса-Яна (абстрактной версии) [16], [20], утверждающей эквивалентность отсутствия асимптотического бесплатного ленча (NAFL) и существования эквивалентной разделяющей меры.

Целью настоящей работы является доказательство аналогичного результата, в котором однако довольно трудно интерпретируемое понятие (NAFL) (использующее замыкания в $*$-слабой топологии на $L^{\infty}$, см. разд. 3.2 для точного определения) заменяется на экономически более убедительное понятие, которое мы называем отсутствием асимптотического бесплатного ленча с исчезающим риском (NAFLVR) и которое полностью соответствует классическому условию отсутствия бесплатного ленча с исчезаюшим риском (NFLVR) для малых рынков, впервые введенному в [5].

Для того, чтобы описать это понятие, рассмотрим пример рынка, состоящего из счетного числа (дисконтированных) активов, моделируемых последовательностью $\mathbb{R}$-значных семимартингалов $\left(S_{t}^{n}\right)_{t \in[0,1]}$, определенных на фильтрованном вероятностном пространстве $\left(\Omega, \mathscr{F}_{t},\left(\mathscr{F}_{t}\right)_{t \in[0,1]}, P\right)$ с временным интервалом $[0,1]$. Так же, как и в работе М. ДеДонно и др. [2], мы определим допустимье обобщенные портфели в большом финансовом рынке как пределы в топологии Эмери последовательностей (равномерно) допустимых портфелей в малых рынках, т.е. таких последовательностей портфелей, отвечающих торговле конечным числом активов, что они равномерно (по $\omega, t$ и $n$ ) ограничены снизу некоторой константой $-\lambda$. В терминологии [2], [3] эти портфели соответствуют портфелям, построенным с помощью так называемых $(\lambda)$-допустимых обобщенньх стратегий (ср. [2, определение 2.5]) и формализуют идею о том, что каждый актив может внести свой вклад, возможно, бесконечно малого веса. Точнее, при рассмотрении обобщенных стратегий мы включаем портфели $Z$, которые обладают тем свойством, что для любого $\varepsilon>0$ существует портфель в малом рынке, для которого расстояние между всеми его приращениями и приращениями $Z$ меньше, чем $\varepsilon$. Это просто следует из определения метрики Эмери (см. (2.1)), в которой два семимартингала (портфеля) близки, если все их приращения близки или, с более финансовой точки 
зрения, если все инвестиции (с ограниченными простыми стратегиями) в разность двух портфелей невелики. Поэтому топология Эмери может рассматриваться, как натуральная топология на множестве процессов капитала портфелей. В частности, есть четкий экономический смысл в том, чтобы включить пределы (в топологии Эмери) портфелей в малых рынках в безарбитражные требования на большом финансовом рынке, как описано в дальнейшем.

В полной аналогии с понятием (NFLVR) в классической ситуации малых финансовых рынков, мы определяем (NAFLVR) как

$$
\bar{C} \cap L_{\geqslant 0}^{\infty}=\{0\},
$$

где $C$ обозначает выпуклый конус ограниченных платежных обязательств, суперреплицируемых (допустимыми обобщенными стратегиями) при цене 0 , т.е. $C=\left(K_{0}-L_{\geqslant 0}^{0}\right) \cap L^{\infty}$, и $K_{0}$ обозначает множество терминальных значений допустимых обобщенных портфелей в момент времени 1. На словах (NAFLVR) означает, что не должно быть никакой последовательности терминальных выплат с допустимыми обобщенными стратегиями, у которых их отрицательные части стремятся к 0 в $L^{\infty}$, в то время как они сходятся почти наверное к неотрицательной случайной величине, которая строго положительна с положительной вероятностью. Также полностью аналогично (NFLVR), (NAFLVR) эквивалентно двум условиям, а именно, отсутствию неограниченной прибыли с ограниченньм риском (NUPBR) и отсутствию арбитража для большого рынка (NA). Условие (NUPBR) означает, что терминальные значения портфелей, построенных из 1-допустимых обобщенных стратегий, ограничены по вероятности, в то время как (NA) требует, чтобы почти наверное неотрицательные терминальные значения допустимых обобщенных портфелей почти наверное равнялись нулю, т.е. $K_{0} \cap L_{\geqslant 0}^{0}=\{0\}$. B сравнении с существующей литературой по большим финансовым рынкам, (NUPBR) эквивалентно отсутствию асимптотического арбитража первого рода (NAA1), которое описывает невозможность получения произвольного богатства с положительной вероятностью, беря произвольно малый (исчезающий) риск (см., например, [9], [10, определение 1] или [14, определение 1.1]). В частности, условие (NAFLVR) отличается от предыдущих понятий асимптотического арбитража более сильным требованием (NA) к большому рынку. Действительно, до сих пор (NA) требовалось только для каждого малого рынка, но не для портфелей, полученных с помощью обобщенных стратегий. Это усиление позволяет получить версию фундаментальной теоремы теории арбитража для больших финансовых рынков, которая до сих пор имелась при описанном выше условии (NAFL). На примере рынка, состоящего из счетного числа активов, она гласит следующее и получается из теоремы 3.2 . 
Теорема 1.1. Пусть $\left(S_{t}^{n}\right)_{t \in[0,1]}$ - последовательность семимартингалов на $\left(\Omega, \mathscr{F},\left(\mathscr{F}_{t}\right), P\right)$. Тогда эквивалентная разделяющая мера $Q \sim P$, m.е. такая мера $Q$, ито $E_{Q}\left[X_{1}\right] \leqslant 0$ для всех $X_{1} \in K_{0}$, существует тогда и только тогда, когда выполнено условие (NAFLVR).

Поэтому (NAFLVR) можно рассматривать как экономически значимое условие «отсутствия арбитража», которое позволяет сделать вывод о существовании разделяющей меры. Ее существование, например, предполагалось и играло решающую роль в работе М. Де Донно и др. [2] по суперрепликации и максимизации полезности в больших финансовых рынках. Мы также считаем, что (NA) есть очень разумное требование для больших финансовых рынков.

Для того, чтобы обеспечить единую трактовку различных финансовых рынков, включающих, например, континуум активов (как в случае рынков облигаций), мы формулируем наши результаты в расширенной версии абстрактных процессов капитала портфелей, введенных в [8]. Основное изменение состоит в ослаблении так называемого свойства конкатенации, которое в настоящей ситуации выполняется только на плотном множестве (по отношению к топологии Эмери) допустимых обобщенных портфелей в большом финансовом рынке. Несмотря на это ослабление, большая часть оригинального доказательства классической фундаментальной теоремы теории арбитража Ф. Делбана и В. Шахермайера [5] может быть перенесена в эту новую ситуацию. Мы адаптируем вариант доказательства из [1], в котором каверзные леммы из оригинального доказательства заменены при помощи определенного свойства ограниченности в топологии Эмери, называемого в литературе свойством предсказуемой равномерной плотности (P-UT).

Оставшаяся часть статьи организована следующим образом: в разд. 2 мы введем модель большого финансового рынка, которая позволяет работать как со счетным, так и с несчетным числом активов. Раздел 2.1 посвящен объяснению точной связи с моделью большого финансового рынка из [2], как намечено выше. Точное определение (NAFLVR) и главный результат, а также связь с (NAFL), формулируются в разд. 3. В разд. 4 мы напоминаем другие понятия «отсутствия (асимптотического) арбитража» и анализируем их взаимосвязи, в то время как в разд. 5 мы показываем, почему альтернативные множества процессов капитала портфелей в больших финансовых рынках не приводят к желаемому результату. В разд. 6 приводится пример, показывающий, что в больших финансовых рынках существование эквивалентной разделяющей меры не обязательно обеспечивает существование эквивалентной $\sigma$-мартингальной меры. В разд. 7 мы приведем доказательство настоящей версии FTAP. Приложение А завершает изложение некоторыми техническими результатами. 
2. Постановка задачи. Мы модифицируем постановку задачи, предложенную Ю. Кабановым в [8], для того, чтобы смоделировать большие финансовые рынки. Точнее, пусть $\left(\Omega, \mathscr{F},\left(\mathscr{F}_{t}\right)_{t \in[0,1]}, P\right)$ - вероятностное пространство с фильтрацией, удовлетворяющее обычным условиям. Пусть $\mathbb{S}$ - пространство всех $\mathbb{R}$-значных семимартингалов $X$ на этом фильтрованном вероятностном пространстве, определенных на $[0,1]$ и стартующих из нуля. Пространство $\mathbb{S}$ снабжено топологией Эмери, определяемой метрикой

$$
d_{\mathbb{S}}\left(X_{1}, X_{2}\right):=\sup _{K \in b \mathscr{E},\|K\|_{\infty} \leqslant 1} E\left[\left|\left(K \bullet\left(X_{1}-X_{2}\right)\right)\right|_{1}^{*} \wedge 1\right]
$$

где $|X|_{1}^{*}=\sup _{t \leqslant 1}\left|X_{t}\right|, b \mathscr{E}$ обозначает множество простых предсказуемых стратегий, т.е. $K$ имеет вид

$$
K=\sum_{i=0}^{n} K_{i} 1_{] \tau_{i}, \tau_{i+1}\right]},
$$

с $n \in \mathbb{N}$, моментами остановки $0=\tau_{0} \leqslant \tau_{1} \leqslant \cdots \leqslant \tau_{n} \leqslant \tau_{n+1}=1$ и $\mathscr{F}_{\tau_{i}}$-измеримыми случайными величинами $K_{i}$. Пространство семимартингалов есть полное метрическое пространство с топологией Эмери, что следует, по существу, из теоремы Бихтелера-Деллашери, см. [6]. Отметим, что М.Эмери определяет метрику с помощью верхней грани по всем ограниченным предсказуемым процессам (см. [6]), а не только по всем простым предсказуемым процессам. Однако, как показано, например, в [17], это приводит к эквивалентной метрике.

Кроме сходимости в топологии Эмери мы также будем иметь дело с равномерной сходимостью по вероятности, которая метризуется метрикой

$$
d(X, Y):=\mathbf{E}\left[|X-Y|_{1}^{*} \wedge 1\right],
$$

и делает пространство càdlàg процессов полным метрическим пространством. Очевидно, топология равномерной сходимости по вероятности слабее топологии Эмери.

Теперь определим модель большого финансового рынка. Пусть $I \subseteq[0, \infty)$ - пространство параметров, которое может быть любым подмножеством, счетным или несчетным. Определим для каждого $n \geqslant 1$ семейство $\mathscr{A}^{n}$ подмножеств $I$, которое содержит ровно $n$ элементов:

$$
\mathscr{A}^{n}=\{\text { некоторые/все подмножества } A \subseteq I, \text { такие, что }|A|=n\},
$$

где $|A|$ обозначает мощность множества $A$. Кроме того, мы предполагаем, что если $A^{1}, A^{2} \in \bigcup_{n \geqslant 1} \mathscr{A}^{n}$, то $A^{1} \cup A^{2} \in \bigcup_{n \geqslant 1} \mathscr{A}^{n}$.

Для каждого $A \in \bigcup_{n \geqslant 1} \mathscr{A}^{n}$ мы определим следующее множество 1-допустимьх прочессов капитала портфелей в малом финансовом рьнке $A$. 
О п р е д е л е н и е 2.1. Для каждого $A \in \bigcup_{n \geqslant 1} \mathscr{A}^{n}$ мы рассмотрим выпуклое множество $\mathscr{X}_{1}^{A} \subset \mathbb{S}$ семимартингалов,

(i) выходящих из 0;

(ii) ограниченных снизу -1 ;

(iii) удовлетворяющих следующему свойству конкатенации: для всех ограниченных, предсказуемых стратегий $H, G \geqslant 0, X, Y \in \mathscr{X}_{1}{ }^{A}$ с $H G=0$ и $Z=(H \bullet X)+(G \bullet Y) \geqslant-1$, выполняется $Z \in \mathscr{X}_{1}^{A}$;

(iv) для $A^{1}, A^{2} \in \bigcup_{n \geqslant 1} \mathscr{A}^{n}$ с $A^{1} \subseteq A^{2}$ справедливо $\mathscr{X}_{1}^{A^{1}} \subset \mathscr{X}_{1}^{A^{2}}$.

Далее мы определяем множество $\mathscr{X}_{1}^{n}$ всех 1-допустимых процессов капитала портфелей, стратегии которых включают максимум $n$ активов (но возможен выбор любых $n$ активов). В самом деле, для каждого $n \geqslant 1$ мы рассмотрим следующее множество $\mathscr{X}_{1}^{n} \subset \mathbb{S}$ семимартингалов:

$$
\mathscr{X}_{1}^{n}=\bigcup_{A \in \mathscr{A}^{n}} \mathscr{X}_{1}^{A}
$$

Заметим, что множества $\mathscr{X}_{1}^{n}$ не являются выпуклыми и не удовлетворяют свойству конкатенации, как в определении 2.1 , потому что в обоих случаях в комбинациях могут быть задействованы $2 n$ активов. Поэтому результат будет лежать в $\mathscr{X}_{1}^{2 n}$, а не в $\mathscr{X}_{1}^{n}$.

Следующие два примера иллюстрируют основные приложения, которые мы имели в виду. Когда мы имеем дело с многомерными семимартингалами и соответствующими многомерными стратегиями, мы используем полужирные буквы, чтобы отличить их от одномерных аналогов.

П р и м е р 2.1 (Приложение 1: Большой финансовый рынок на одном вероятностном пространстве). Также, как и во введении или, например, в [2], рассмотрим большой финансовый рынок, который моделируется последовательностью семимартингалов $\left(S_{t}^{n}\right)_{t \in[0,1], n \in \mathbb{N}}$, заданных на вероятностном пространстве с фильтрацией $\left(\Omega, \mathscr{F},\left(\mathscr{F}_{t}\right)_{t \in[0,1]}, P\right)$.

Наша постановка охватывает эту модель. Действительно, выберем $I=\mathbb{N}$ и определим $\mathscr{A}^{n}=\left\{A^{n}\right\}$ как одноэлементное множество, содержащее только множество $A^{n}=\{1, \ldots, n\}$. Множество $\mathscr{X}_{1}^{n}=\mathscr{X}_{1}^{A^{n}}$ тогда состоит из всех стохастических интегралов $\left(\mathbf{H}^{n} \bullet \mathbf{S}^{n}\right)$, где $\mathbf{S}^{n}=$ $\left(S^{1}, \ldots, S^{n}\right)$ и $\mathbf{H}^{n}$ - это $n$-мерные, предсказуемые, $\mathbf{S}^{n}$-интегрируемые и 1-допустимые стратегии.

П р и м е р 2.2 (Приложение 2: Рынки на основе континуума активов, такие как рынки облигаций). Предположим, что нам дан континуум торгуемых активов, стоимость которых выражена в некоторых единицах (numéraire). Эти активы даны как семимартингалы $\left(S_{t}^{\alpha}\right)_{0 \leqslant t \leqslant 1}$, $\alpha \in I$, заданные на $\left(\Omega, \mathscr{F},\left(\mathscr{F}_{t}\right)_{0 \leqslant t \leqslant 1}, P\right)$. Можно, например, выбрать параметрическое множество $I=\left[0, T^{*}\right], T^{*} \leqslant \infty$, где $\alpha \in I$ может трактоваться как срок погашения облигации. Здесь мы выбираем $\mathscr{A}^{n}$ как семейство всех подмножеств $A \subseteq I$, таких, что $|A|=n$. 
Для $A \in \mathscr{A}^{n}$, где $A=\left\{\alpha_{1}, \ldots, \alpha_{n}\right\}$, для $\alpha_{1}, \ldots, \alpha_{n} \in I$, множество $\mathscr{X}_{1}^{A}$ задается как

$$
\mathscr{X}_{1}^{A}=\left\{\left(\mathbf{H}^{A} \bullet \mathbf{S}^{A}\right)_{0 \leqslant t \leqslant T}, \mathbf{H}^{A}-1 \text {-допустим }\right\}
$$

где $\mathbf{S}^{A}=\left(S^{\alpha_{1}}, \ldots, S^{\alpha_{n}}\right), \mathbf{H}^{A}-n$-мерный, предсказуемый 1-допустимый интегрант для $\mathbf{S}^{A}$. Очевидно, что множества $\mathscr{X}_{1}^{A}$ удовлетворяют требованиям определения 2.1. Множества $\mathscr{X}_{1}^{n}, n \geqslant 1$, выражают тот факт, что можно торговать любым конечным числом активов (но допускается каждый конечный набор из несчетного количества активов $\left.S^{\alpha}, \alpha \in I\right)$.

Продолжая общую постановку, определим теперь множества $\mathscr{X}$ (и $\mathscr{X}_{1}$ соответственно), которые заменяют соответствующие множества из [8], и называются (1-) допустимыми обобщенными процессами капитала портфелей в большом финансовом рынке.

О п р е д е л е н и е 2.2. (i) Рассмотрим множество $\mathscr{X}_{1}={\overline{\bigcup_{n \geqslant 1} \mathscr{X}_{1}^{n}}}^{S}$, где $\overline{(\cdot)}$ обозначает замыкание в топологии Эмери. Элементы $\mathscr{X}_{1}$ называются 1-допустимыми обобщенными процессами капитала портфелей в большом финансовом рынке.

(ii) Обозначим через $\mathscr{X}$ множество $\mathscr{X}:=\cup_{\lambda>0} \lambda \mathscr{X}_{1}$ и назовем его элементы допустимьми обобщенньми процессами капитала портфелей в большом финансовом рынке.

(iii) Обозначим через $K_{0}$, соответственно $K_{0}^{1}$, множество значений элементов из $\mathscr{X}$, соответственно из $\mathscr{X}_{1}$, в конечный момент $T=1$.

3 а м е ч а н и е 2.1. В примере 2.2 допустимые обобщенные процессы капитала портфелей также включают портфели $Z$, в которых комбинации облигаций в портфеле может изменяться с течением времени настолько долго, пока это изменение не слишком «большое». Например, если $Z$ - это семимартингал (соответствующий такому портфелю), и если существует последовательность моментов остановки $\left(\tau_{k}\right)_{k \in \mathbb{N}}$, сходящаяся п.н. к 1 так, что для каждого $k \in \mathbb{N}$ можно записать $Z^{\tau_{k}}$ как

$$
Z_{1}^{\tau_{k}}=\left(\mathbf{H}^{A_{k}} \bullet \mathbf{S}^{A_{k}}\right)_{1 \wedge \tau_{k}}
$$

для некоторой $\lambda$-допустимой стратегии $\mathbf{H}^{A_{k}}$, которая инвестирует в облигации, соответствующие множеству индексов $A_{k}$, до времени $\tau_{k}$.

Например, портфель $Z$ может быть построен по стратегии, которая всегда инвестирует в конечное число активов, но в определенные моменты остановки состав портфеля может быть изменен, чтобы включить новое конечное число активов.

Лемма 2.1 (Свойство конкатенации). Плотное в топологии Эмери подмножество $\bigcup_{n \geqslant 1} \mathscr{X}_{1}^{n}$ множества $\mathscr{X}_{1}$ удовлетворяет следующему свойству конкатенации: пусть $X, Y \in \bigcup_{n \geqslant 1} \mathscr{X}_{1}^{n}$, тогда существует 
такое $A \in \bigcup_{n \geqslant 1} \mathscr{A}^{n}$, что $X, Y \in \mathscr{X}_{1}^{A}$. Для всех ограниченньх, предсказуемьх стратегий $H, G \geqslant 0$, с $H G=0$ и таких, ито $Z=(H \bullet X)+(G \bullet$ $Y) \geqslant-1$, выполнено $Z \in \mathscr{X}_{1}^{A} u$, следовательно, $Z \in \bigcup_{n \geqslant 1} \mathscr{X}_{1}^{n}$.

Д о к а з а т е л ь с т в о. Если $X, Y \in \bigcup_{n \geqslant 1} \mathscr{X}_{1}^{n}=\bigcup_{n \geqslant 1} \bigcup_{A \in \mathscr{A}_{n}} \mathscr{X}_{1}^{A}$, то существуют $A^{1}, A^{2} \subseteq I \mathrm{c}\left|A^{1}\right|=n$ и $\left|A^{2}\right|=m$, такие, что $X \in \mathscr{X}_{1}^{A^{1}}$ и $Y \in \mathscr{X}_{1}^{A^{2}}$. Пусть $A=A^{1} \cup A^{2}$. Тогда $A \in \bigcup_{n \geqslant 1} \mathscr{A}^{n}$ и $X, Y \in \mathscr{X}_{1}^{A}$, где $A$ содержит не более $n+m$ элементов. Следовательно, по свойству конкатенации для множества $\mathscr{X}_{1}^{A}$ (см. определение 2.1) мы получаем для $H$ и $G$ из формулировки леммы, что для $Z=(H \bullet X)+(G \bullet Y)$,

$$
Z \in \mathscr{X}_{1}^{A} \subseteq \bigcup_{n \geqslant 1} \mathscr{X}_{1}^{n}
$$

Лемма 2.1 доказана.

3 а м е ч а н и е 2.2. Обратите внимание, что у нас есть свойство конкатенации только на плотном подмножестве множества всех допустимых портфелей, т.е. мы не можем непосредственно применить рассуждения из [8] или [1]. Нет универсального способа, чтобы распространить свойство конкатенации в этой бесконечномерной постановке на замыкание в топологии Эмери. Математически говоря, цель этой работы показать, что этого все еще достаточно для всех выводов.

\section{1. Связь с литературой по обобщенным торговым стра-} тегиям в большом финансовом рынке. Мы сосредоточимся здесь на ситуации большого финансового рынка, рассмотренной М. Де Донно и др. в [2], которая описывается в примере 2.2. Как уже отмечалось там, множество $\mathscr{X}_{1}^{n}$, соответствующее 1-допустимым процессам капитала портфелей в малом финансовом рынке $n$, задается здесь следующим множеством стохастических интегралов по $n$-мерному семимартингалу $\mathbf{S}^{n}=\left(S^{1}, \ldots, S^{n}\right)$ :

$$
\mathscr{X}_{1}^{n}=\left\{\left(\mathbf{H} \bullet \mathbf{S}^{n}\right) \mid \mathbf{H} \in \mathscr{H}_{1}^{n}\right\}
$$

C

$$
\begin{gathered}
\mathscr{H}_{\lambda}^{n}=\left\{\mathbf{H} \mid \mathbb{R}^{n} \text {-значный, предсказуемый, } \mathbf{S}^{n}\right. \text {-интегрируемый и } \\
\lambda \text {-допустимый процесс }\} .
\end{gathered}
$$

Как обычно $\lambda$-допустимость означает, что $\left(\mathbf{H} \bullet \mathbf{S}^{n}\right)_{t} \geqslant-\lambda$ для всех $t \in$ $[0,1]$.

В работе [2] переход от последовательности малых финансовых рынков к модели большого финансового рынка достигается с помощью обобщенной теории стохастического интегрирования по последовательности семимартингалов. Как уже упоминалось во введении, соответствующие 
интегранты называются обобщенными стратегиями. Это понятие формализует идею портфеля, в котором каждый актив вносит вклад, возможно, с бесконечно малым весом. Как мы увидим в предложении 2.1, множество $\mathscr{X}_{1}$ из определения 2.2 точно соответствует стохастическим интегралам с 1-допустимьми обобщенными стратегиями, определенными ниже и первоначально рассмотренными в [3]. В следующем определении $\mathbb{S}-\lim$ обозначает предел в топологии Эмери.

О п р е д е л е н и е 2.3. (i) Для каждого $n \in \mathbb{N}$, пусть $\mathbf{H}^{n}-\mathbb{R}^{n}$ значный, предсказуемый, $\mathbf{S}^{n}$-интегрируемый процесс. Последовательность $\left(\mathbf{H}^{n}\right)_{n \in \mathbb{N}}$ называется обобщенной стратегией, если $\left(\mathbf{H}^{n} \bullet \mathbf{S}^{n}\right)$ сходится в топологии Эмери к семимартингалу

$$
Z:=\mathbb{S}-\lim \left(\mathbf{H}^{n} \bullet \mathbf{S}^{n}\right),
$$

который называется обобщенным стохастическим интегралом.

(ii) Пусть $\lambda>0$. Обобщенная стратегия $\left(\mathbf{H}^{n}\right)$ называется $\lambda$ допустимой, если каждый элемент аппроксимирующей последовательности $\mathbf{H}^{n} \lambda$-допустим.

Предложение 2.1. Рассмотрим следующее множество

$$
\mathscr{Z}_{1}=\left\{\mathbb{S}-\lim \left(\mathbf{H}^{n} \bullet \mathbf{S}^{n}\right) \mid\left(\mathbf{H}^{n}\right)-1 \text {-допустимая обобщенная стратегия }\right\} .
$$

Тогда $\mathscr{Z}_{1}=\mathscr{X}_{1}$, где $\mathscr{X}_{1}$ задается в определении 2.2 с $\mathscr{X}_{1}^{n}$, определенньм в (2.5).

Д о к а з а т е л ь с т в о. Вложение $\mathscr{Z}_{1} \subseteq{\overline{\bigcup_{n \geqslant 1} \mathscr{X}_{1}^{n}}}^{\mathbb{S}}=\mathscr{X}_{1}$ очевидно из определения 1-допустимых обобщенных стратегий.

Обратно, пусть $X \in \mathscr{X}_{1}$. Тогда существует некоторая последовательность $X^{k} \in \bigcup_{n \geqslant 1} \mathscr{X}_{1}^{n}$, такая, что $X^{k} \rightarrow X$ в топологии Эмери. Так как каждый $X^{k}$ лежит в некотором $\mathscr{X}_{1}^{n_{k}}$, то справедливо $X^{k}=\mathbf{H}^{n_{k}} \bullet \mathbf{S}^{n_{k}}$ для некоторого $\mathbf{H}^{n_{k}} \in \mathscr{H}_{1}^{n_{k}}$, что доказывает $\mathscr{X}_{1} \subseteq \mathscr{Z}_{1}$. Предложение 2.1 доказано.

3 а м е ч а н и е 2.2. Взаимно однозначное соответствие между $\mathscr{Z}_{1}$ и $\mathscr{X}_{1}$ в постановке [2] послужило для нас мотивацией, чтобы определить $\mathscr{X}_{1}$ как $\overline{\bigcup_{n \geqslant 1} \mathscr{X}_{1}^{n}} \mathbb{S}$.

3. Основные понятия и результаты. Для того, чтобы ввести понятие отсутствия асимптотического арбитража, мы должны использовать следующие выпуклые конусы:

$$
C_{0}:=K_{0}-L_{\geqslant 0}^{0}, \quad C:=C_{0} \cap L^{\infty}
$$

По полной аналогии с условием отсутствия бесплатного ленча с исчезающим риском (NFLVR), мы определим 
(NAFLVR): Множество $\mathscr{X}$ удовлетворяет условию отсутствия асимптотического бесплатного ленча с исчезающим риском, если

$$
\bar{C} \cap L_{\geqslant 0}^{\infty}=\{0\}
$$

где $\bar{C}$ обозначает замыкание по норме в $L^{\infty}$.

3.1. Фундаментальная теорема теории арбитража для больших финансовых рынков. Прежде чем сформулировать наш главный результат, давайте вспомним понятие эквивалентной разделяющей меры.

О п р е д е л е н и е 3.1. Множество $\mathscr{X}$ удовлетворяет условию (ESM) (существования эквивалентной разделяющей мерь), если существует эквивалентная мера $Q \sim P$, такая, что $E_{Q}\left[X_{1}\right] \leqslant 0$ для всех $X \in \mathscr{X}$.

Заметим, что при выполнении условия

$$
\bar{C}^{*} \cap L_{\geqslant 0}^{\infty}=\{0\},
$$

где $\bar{C}^{*}$ обозначает *-слабое замыкание в $L^{\infty},(\mathrm{ESM})$ является следствием теоремы Крепса-Яна [16], [20], которая, в свою очередь, следует из теоремы Хана-Банаха. Условие (NFL) является классическим условием отсутствия бесплатного ленча (NFL) для абстрактного множества $C$.

Очевидно, что $(\mathrm{NFL}) \Rightarrow(\mathrm{NAFLVR})$. Целью является показать обратное следствие, т.е. справедливо следующее утверждение.

Tеорема 3.1. Если вьлполнено условие (NAFLVR), то $C=\bar{C}^{*}$, т.е. конус $C$ является *-слабо залкнутыл, и выполняется условие (NFL).

Этот результат доказан в разд. 7, где мы адаптируем методы, используемые в [1], к текущим условиям больших финансовых рынков. Так же, как и при классических условиях, это приведет к следующей теореме 3.2, которая является версией фундаментальной теоремы теории арбитража для больших финансовых рынков.

Теорема 3.2 (Фундаментальная теорема теории арбитража для больших финансовых рынков). Имеет место (NAFLVR) $\Leftrightarrow$ (ESM).

Заметим, что соответствующий результат для рынка, состоящего из конечного множества активов, был впервые доказан Делбаном и Шахермайером в [5]. Кабанов [8] нашел более абстрактные условия для допустимых процессов капитала портфелей, указанные в определении 2.1, для которых может быть применено доказательство из [5].

3.2. Связь условия отсутствия асимптотического бесплатного ленча (NAFL) и соответствующей фундаментальной теоремы теории арбитража. В этом разделе мы обсудим связь (NAFLVR) с идеей отсутствия асимптотического бесплатного ленча 
(NAFL), см. [13]. Здесь мы снова попадаем в ситуацию примера 2.2. Пусть

$$
\mathscr{K}_{0}=\left\{X_{1}, \text { где } X \in \bigcup_{\lambda>0} \bigcup_{n \geqslant 1} \lambda \mathscr{X}_{1}^{n}=\bigcup_{n \geqslant 1} \mathscr{X}^{n}\right\},
$$

где $\mathscr{X}_{1}^{n}$ заданы как в определении 2.1 (в конкретном примере 2.1 ), и $\mathscr{X}^{n}$ заданы как в замечании 2.3. Множество $\mathscr{K}_{0}$ состоит из конечных значений допустимых портфелей на всех малых рынках $n$, но без замыкания в топологии Эмери. Это соответствует обычным условиям для больших финансовых рынков при дополнительном предположении, что все процессы заданы на едином вероятностном пространстве $(\Omega, \mathscr{F}, P)$, см., например, [9], [14], [13]. Определим

$$
\mathscr{C}=\left(\mathscr{K}_{0}-L_{\geqslant 0}^{0}\right) \cap L^{\infty} .
$$

Тогда условие (NAFL) следует понимать следующим образом.

(NAFL): Множество $\mathscr{C}$ удовлетворяет условию отсутствия асимптотического бесплатного ленча, если

$$
\overline{\mathscr{C}}^{*} \cap L_{\geqslant 0}^{\infty}=\{0\},
$$

где $\overline{\mathscr{C}}^{*}$ означает *-слабое замыкание в $L^{\infty}$.

Для того, чтобы сравнить FTAP из [13] с теоремой 3.2 выше, мы должны определить подходящие условия (ESM).

О п р е д е л е н и е 3.2. Множество $\bigcup_{\lambda>0} \bigcup_{n \geqslant 1} \lambda \mathscr{X}_{1}^{n}$ удовлетворяет свойству (ESM'), если существует эквивалентная мера $Q \sim P$, такая, что $E_{Q}\left[X_{1}\right] \leqslant 0$ для всех $X \in \bigcup_{\lambda>0} \bigcup_{n \geqslant 1} \lambda \mathscr{X}_{1}^{n}$.

В [13] следующая фундаментальная теорема теории арбитража для больших финансовых рынков была доказана в общей ситуации для последовательностей возможно различных вероятностных пространств $\Omega^{n}$ (где условия (NAFL) выглядят более техническими, но соответствуют нашему определению (NAFL), если все $\Omega^{n}$ совпадают). В нашем случае, когда все $\Omega^{n}$ совпадают, это также может быть выведено из (абстрактной версии) теоремы Крепса-Яна [16], [20].

Теорема 3.3. Имеет место: (NAFL) $\Leftrightarrow\left(\mathrm{ESM}^{\prime}\right)$.

Сейчас мы покажем, что теоремы 3.2 и 3.3 эквивалентны ввиду того, что имеется точное соотношение между множествами $C$ и $\mathscr{C}$. Чтобы показать это, используем поляры множеств $C$ и $\mathscr{C}$. Определим

$$
\mathscr{M}=\left\{Q \ll P: E_{Q}[f] \leqslant 0 \text { для всех } f \in C\right\} .
$$

Легко увидеть, что $\mathscr{M}$ является множеством абсолютно непрерывных разделяющих мер для $\mathscr{X}$, т.е.

$$
\mathscr{M}=\left\{Q \ll P: E_{Q}\left[X_{1}\right] \leqslant 0 \text { для всех } X \in \mathscr{X}\right\} .
$$


Более того, сейчас мы увидим, что множество $\mathscr{M}$ совпадает с множеством абсолютно непрерывных разделяющих мер в смысле $\left(\mathrm{ESM}^{\prime}\right)$.

Лемма 3.1. Мера $Q \in \mathscr{M}$ тогда и только тогда, когда $Q-$ - абсолютно непрерьвная разделяющая мера для $\bigcup_{\lambda>0} \bigcup_{n \geqslant 1} \lambda \mathscr{X}_{1}^{n}$.

Д о к а з а т е л ь с т в о. Пусть $Q$ удовлетворяет условиям из определения $\left(\mathrm{ESM}^{\prime}\right)$. В частности, $E_{Q}\left[X_{1}\right] \leqslant 0$ для всех $X \in \bigcup_{n \geqslant 1} \mathscr{X}_{1}^{n}$. Мы должны показать, что $E_{Q}\left[Y_{1}\right] \leqslant 0$ для $Y \in \mathscr{X}_{1}$. По определению существует последовательность процессов $X^{k} \in \bigcup_{n \geqslant 1} \mathscr{X}_{1}^{n}$, такая, что $X^{k} \rightarrow Y$ в топологии Эмери. Это влечет $X_{1}^{k} \rightarrow Y_{1}$ по вероятности. По предположению $X_{1}^{k} \geqslant-1$, для всех $k$, и из условия $\left(\mathrm{ESM}^{\prime}\right)$ следует, что $E_{Q}\left[X_{1}^{k}\right] \leqslant 0$, для всех $k$. Из леммы Фату вытекает, что $E_{Q}\left[Y_{1}\right] \leqslant 0$. В обратную сторону утверждение очевидно, так как

$$
\bigcup_{\lambda>0} \bigcup_{n \geqslant 1} \lambda \mathscr{X}_{1}^{n} \subseteq \bigcup_{\lambda>0} \lambda \mathscr{X}_{1}=\mathscr{X} .
$$

Лемма 3.1 доказана.

Рассмотрим двойственность $\left(L^{\infty}, L^{1}\right)$ с билинейной формой $E_{P}[f g]$ для $f \in L^{\infty}$ и $g \in L^{1}$. Пусть $B$ будет любым подмножеством из $L^{\infty}$. Рассмотрим поляру $B^{\circ}$ множества $B$; как обычно,

$$
B^{\circ}=\left\{g \in L^{1}:\left|E_{P}[f g]\right| \leqslant 1 \text { для всех } f \in B\right\} .
$$

Если $B$ является выпуклым конусом (что имеет место как в случае $C$, так и в случае $\mathscr{C})$, то поляра задается как

$$
B^{\circ}=\left\{g \in L^{1}: E_{P}[f g] \leqslant 0 \text { для всех } f \in B\right\} .
$$

Сейчас мы приведем соотношение между множествами $C$ и $\mathscr{C}$. Оказывается, что при выполнении (NAFLVR) множество $C$ является в точности $*$-слабым замыканием множества $\mathscr{C}$. Это значит, что замыкание в топологии Эмери в определении множества $\mathscr{X}_{1}$ эквивалентно $*$-слабому замыканию множества $\mathscr{C}$ (которое задается также как $C$, но без замыкания в топологии Эмери). Так что в итоге условия (NAFL) и (NAFLVR) совпадают.

Tеорема 3.4. Предположим что выполняется условие (NAFLVR). Тогда $C=\overline{\mathscr{C}}^{*}$.

Д о к а з а т е л ь с т в о. Мы покажем, что

$$
C^{\circ}=\mathscr{C}^{\circ}=\bigcup_{\alpha \geqslant 0}(\alpha \mathscr{M}) .
$$

В самом деле, каждый элемент $g$ в правой части удовлетворяет $E_{P}[f g] \leqslant 0$, для каждого $f$, принадлежащего $C$ (или $\mathscr{C}$ (см. лемму 3.1 ). 
Поэтому $\bigcup_{\alpha \geqslant 0}(\alpha \mathscr{M}) \subseteq C^{\circ}$ (или $\left.\mathscr{C}^{\circ}\right)$. С другой стороны, пусть $g \in C^{\circ}$ или $\mathscr{C}^{\circ}$. В обоих случаях, так как

$$
-L_{\geqslant 0}^{\infty} \subseteq C \quad(\text { или } \mathscr{C})
$$

мы тут же получаем, что $g \geqslant 0$. Предполагая нетривиальный случай, когда $P(g>0)>0$, зададим $Q$ как $d Q / d P=g / E_{P}[g]$. Тогда это абсолютно непрерывная мера, такая, что $E_{Q}[f] \leqslant 0$ для всех $f \in C$ (или $f \in \mathscr{C}$ ), соответственно. Это доказывает приведенное выше утверждение.

Мы знаем, что при выполнении условия (NAFLVR) $C$ является слабо-ж-замкнутым подмножеством $L^{\infty}$. Более того, $C$ является выпуклым конусом. По биполярной теореме биполяра $C^{\circ \circ}$ является слабым*-замыканием $C$, см. [19]. Поэтому

$$
C^{\circ \circ}=C
$$

С другой стороны, $\mathscr{C}$ также является выпуклым конусом. Поэтому, снова по биполярной теореме,

$$
\mathscr{C}^{\circ \circ}=\overline{\mathscr{C}}^{*} .
$$

По причинам, указанным выше, $C^{\circ}=\mathscr{C}^{\circ}$, откуда следует, что $C=\overline{\mathscr{C}}^{*}$. Теорема 3.4 доказана.

3.3. Связь с рынком облигаций из работы [15]. В предыдущей статье [15], рассматривалась частная ситуация бездефолтных рынков облигаций со всеми сроками погашения до конечного временного горизонта $T^{*}$ и было выведено условие, которое позволяет вывести (NFL) для рынков, порожденных самофинансируемыми инвестициями в конечное число облигаций (выбирая облигацию со сроком погашения, равным $T^{*}$, в качестве масштаба цен на рынке). Это также большой финансовый рынок с несчетным количеством активов. Тем не менее, это условие включает только счетный, плотный (большой финансовый) подрынок, т.е. подмножество облигаций (включающее облигацию со сроком погашения $\left.T^{*}\right)$, сроки погашения которых плотны на интервале $\left[0, T^{*}\right]$. А именно, предполагается, что классическое условие (NAFL) выполняется для этих подрынков, и, что более важно, предполагается, что равномерная версия непрерывности справа цен облигаций (по отношению к сроку погашения) выполняется для всего рынка. Это позволяет (в локально ограниченном случае, как в работе [15]) сделать вывод о существовании эквивалентной локально мартингальной меры для всего дисконтированного рынка облигаций, что в свою очередь означает выполнение условия (NAFLVR) или (NFL) на нашем большом финансовом рынке. Поэтому кажущееся абстрактным условие (NAFLVR) при многих обстоятельствах может быть выведено из условий для счетных подрынков при определенных условиях непрерывности. 
4. NA, NUPBR и NAA1. В этом разделе мы напомним перечисленные в названии понятия не (асимптотического) арбитража (из литературы) и проанализируем их взаимосвязи, а также докажем в предложении 4.2 следующее утверждение, которое понадобится для доказательства теоремы 3.1 в разд. 7 :

$$
(\mathrm{NAFLVR}) \Leftrightarrow(\mathrm{NUPBR})+(\mathrm{NA}) .
$$

Мы начнем с условия отсутствия арбитража (NA), которое определяется аналогично малым рынкам. В контексте больших финансовых рынков оно означает, что почти наверное неотрицательные конечные значения допустимых обобщенных портфелей должны равняться нулю почти наверное, что выражается формулами в следующем виде:

(NA): Говорят, что множество $\mathscr{X}_{1}$ удовлетворяет условию отсутствия арбитража, если

$$
K_{0} \cap L_{\geqslant 0}^{0}=\{0\},
$$

что эквивалентно тому, что $C_{0} \cap L_{\geqslant 0}^{0}=\{0\}$ и

$$
C \cap L_{\geqslant 0}^{\infty}=\{0\}
$$

Хорошо известно, что, если на малом финансовом рынке выполняется условие (NA) и конечное значение портфеля ограничено снизу константой, то и весь процесс капитала портфеля ограничен снизу той же константой. Следующая лемма переносит это свойство на нашу постановку для больших финансовых рынков и будет использоваться в доказательстве теоремы 3.1. Доказательство, тем не менее, немного сложнее, чем в случае малых рынков.

Лемма 4.1. Пусть $Y \in \mathscr{X}$. Если условие (NA) выполняется и $Y_{1} \geqslant-1$, mo $Y \in \mathscr{X}_{1}$.

Д о к а з а т е л с с т о. Мы покажем, что $Y \in \mathscr{X}_{1}$. По предположению $Y \in \mathscr{X}=\bigcup_{\lambda>0} \lambda \mathscr{X}_{1}$, и можно выбрать $\lambda:=\sup _{t \in[0,1]} Y_{t}^{-}>0$, такое, что $Y=\lambda Z$ с $Z \in \mathscr{X}_{1}$. Предположим, что $Y \notin \mathscr{X}_{1}$ и $\lambda>1$ (иначе доказывать нечего).

Тогда существуют $t \in[0,1), \alpha>0$ и $1>\varepsilon>0$ с $\lambda-\varepsilon>1$, такие, что

$$
P\left(Y_{t} \leqslant-(\lambda-\varepsilon)\right)=\alpha>0 .
$$

Пусть $\delta=\lambda-\varepsilon-1>0$. Положим $D=\left\{Y_{t} \leqslant-(\lambda-\varepsilon)\right\}$. Определим простой предсказуемый интегрант $H_{u}=\mathbb{I}_{D} \mathbb{1}_{t, 1]}$. Тогда

$$
(H \bullet Y)_{1}=\mathbb{I}_{D}\left(Y_{1}-Y_{t}\right) \geqslant \mathbb{I}_{D}(-1+\lambda-\varepsilon)=\delta \mathbb{I}_{D},
$$

поэтому $(H \bullet Y)_{1} \geqslant 0$ п.н. и $P\left((H \bullet Y)_{1} \geqslant \delta\right)=\alpha>0$. 
Наш следующий шаг - показать, что $(H \bullet Y) \in \mathscr{X}$, тогда мы придем к противоречию с (NA). Мы покажем, что $(H \bullet Y)$ лежит в $\mathscr{X}_{1}$. В самом деле, по определению $\mathscr{X}_{1}$, существует последовательность $Z^{k} \in \bigcup_{n \geqslant 1} \mathscr{X}_{1}^{n}$, такая, что $Z^{k} \rightarrow Z$ в топологии Эмери. Тогда существуют конечные подмножества $A^{k}$ в $I$ с $Z^{k} \in \mathscr{X}_{1}^{A^{k}}$. Более того, очевидно, $Y^{k}:=\lambda Z^{k}$ сходится в топологии Эмери к $Y=\lambda Z$. Легко видеть, что

$$
\left(H \bullet Y^{k}\right) \rightarrow(H \bullet Y)
$$

в топологии Эмери, так как для каждого $K \in b \mathscr{E},\|K\|_{\infty} \leqslant 1$, имеем

$$
\left(K \bullet\left(\left(H \bullet Y^{k}\right)-(H \bullet Y)\right)\right)=\left(K H \bullet\left(Y^{k}-Y\right)\right)
$$

и $K H \in b \mathscr{E},\|K H\|_{\infty} \leqslant 1$.

Тем не менее, мы не можем сказать, что $\left(H \bullet Y^{k}\right) \in \mathscr{X}_{1}^{A^{k}} \subseteq \bigcup_{n \geqslant 1} \mathscr{X}_{1}^{n}$, потому что $\left(H \bullet Y^{k}\right)$ может не превосходить -1 . Поэтому мы аппроксимируем $H$ с помощью простых предсказуемых интегрантов $H^{k}=$ $\mathbb{I}_{D^{k}} \mathbb{I}_{] t, 1]}$, где

$$
D^{k}=\left\{Y_{t}^{k} \leqslant-(\lambda-\varepsilon)+2^{-k}\right\} .
$$

Так как $Y^{k} \rightarrow Y$ в топологии Эмери, то, в частности, $Y_{t}^{k} \rightarrow Y_{t}$ по вероятности и, значит, $\mathbb{I}_{D^{k}} \rightarrow \mathbb{I}_{D}$ по вероятности. Более того, $H^{k} \geqslant 0$ и

$$
\left(H^{k} \bullet Y^{k}\right)_{u}=\mathbb{I}_{D^{k}}\left(Y_{u}^{k}-Y_{t}^{k}\right) \geqslant \mathbb{I}_{D^{k}}\left(-\lambda+\lambda-\varepsilon-2^{-k}\right) \geqslant-\varepsilon \geqslant-1
$$

для каждого $u \geqslant t$. Поэтому, применяя свойство конкатенации из леммы 2.1, получаем, что $\left(H^{k} \bullet Y^{k}\right) \in \mathscr{X}_{1}^{A^{k}} \subseteq \bigcup_{n \geqslant 1} \mathscr{X}_{1}^{n}$.

Наконец покажем, что

$$
d_{\mathbb{S}}\left(\left(H^{k} \bullet Y^{k}\right),\left(H \bullet Y^{k}\right)\right) \rightarrow 0
$$

для $k \rightarrow \infty$. В самом деле, пусть $J^{k}=H^{k}-H$, тогда $J^{k} \neq 0$ только на множестве $E^{k}=D \backslash D^{k} \cup D^{k} \backslash D$, где $P\left(E^{k}\right) \rightarrow 0$ для $k \rightarrow \infty$. Для каждого $K \in b \mathscr{E},\|K\|_{\infty} \leqslant 1$ и $u \geqslant t$, мы получаем, что

$$
\begin{aligned}
\left(K \bullet\left(\left(H^{k} \bullet Y^{k}\right)-\left(H \bullet Y^{k}\right)\right)\right)_{u} & =\left(\left(H^{k}-H\right) \bullet\left(K \bullet Y^{k}\right)\right)_{u} \\
& =\left(\mathbb{I}_{D^{k}}-\mathbb{I}_{D}\right)\left(\left(K \bullet Y^{k}\right)_{u}-\left(K \bullet Y^{k}\right)_{t}\right) .
\end{aligned}
$$

Поэтому для $g_{u}^{k}=\left(K \bullet Y^{k}\right)_{u}-\left(K \bullet Y^{k}\right)_{t}$

$$
\begin{aligned}
\sup _{K \in b \mathscr{E},\|K\|_{\infty} \leqslant 1} E\left[\left|K \bullet\left(\left(H^{k} \bullet Y^{k}\right)-\left(H \bullet Y^{k}\right)\right)\right|_{1}^{*} \wedge 1\right] \\
\leqslant \sup _{K \in b \mathscr{E},\|K\|_{\infty} \leqslant 1} E\left[\left|\mathbb{I}_{D^{k}}-\mathbb{I}_{D}\right|\left(\sup _{u \in[0,1]}\left|g_{u}^{k}\right| \wedge 1\right)\right] \\
\leqslant E\left[\left|\mathbb{I}_{D^{k}}-\mathbb{I}_{D}\right| \sup _{K \in b \mathscr{E},\|K\|_{\infty} \leqslant 1}\left(\sup _{u \in[0,1]}\left|g_{u}^{k}\right| \wedge 1\right)\right] .
\end{aligned}
$$


Но так как $\left|\mathbb{I}_{D^{k}}-\mathbb{I}_{D}\right|=\mathbb{I}_{E^{k}} \rightarrow 0$ по вероятности и так как все члены в (4.3) ограничены 1 , мы получаем с помощью теоремы о мажорируемой сходимости, что ожидаемое значение в (4.3) сходится к 0. Отсюда следует, что $d_{\mathbb{S}}\left(\left(H^{k} \bullet Y^{k}\right),\left(H \bullet Y^{k}\right)\right) \rightarrow 0$.

Это завершает доказательство: мы нашли $\left(H^{k} \bullet Y^{k}\right) \in \bigcup_{n \geqslant 1} \mathscr{X}_{1}^{n}$ с $\left(H^{k} \bullet Y^{k}\right) \rightarrow(H \bullet Y)$ в топологии Эмери, и, поэтому, $(H \bullet Y) \in \mathscr{X}_{1}$ противоречит (NA). Лемма 4.1 доказана.

Сейчас мы введем условия (NAA1) и (NUPBR), которые окажутся эквивалентными.

О п р е д е л е н и е 4.1. Асимптотический арбитраж первого порядка (AА1) существует, если существуют некоторое $\alpha>0$ и последовательности $\varepsilon_{k} \rightarrow 0, c_{k} \rightarrow \infty$ и $X^{k}$, такие, что для каждого $k \in \mathbb{N}$

(i) $X^{k} \in \varepsilon_{k} \bigcup_{n \geqslant 1} \mathscr{X}_{1}^{n}$,

(ii) $P\left[X^{k} \geqslant c_{k}\right] \geqslant \alpha$,

(NAA1): Условие отсутствия асимптотического арбитража первого рода выполняется, если не существует (АA1).

(NUPBR): Говорят что множество $\mathscr{X}_{1}$ удовлетворяет условию отсутствия неограниченной прибыли с ограниченным риском, если $K_{0}^{1}$ ограниченное подмножество в $L^{0}$.

Арбитраж первого рода, который впервые появился под этим названием в [7], был введен в контексте больших финансовых рынков Кабановым и Крамковым [9, определение 1]. Позже Кардарас и Каратзас [11] использовали этот термин в контексте классических малых финансовых рынков. В их постановке показано, что условие (NA1) эквивалентно (соответсвующему понятию) NUPBR. Следующее предложение устанавливает аналогичный результат в случае больших финансовых рынков.

Предложение 4.1. Имеет место: (NAA1) $\Leftrightarrow$ (NUPBR).

Д о к а з а т ельст в о. Сначала докажем, что (NUPBR) $\Rightarrow$ (NAA1). Предположим, от противного, существование (AA1). Тогда существует последовательность $X^{k}$, как в определении 4.1. Для $Y^{k}=X^{k} / \varepsilon_{k} \in \bigcup_{n \geqslant 1} \mathscr{X}_{1}^{n}$ имеем для $k \in \mathbb{N}$

$$
P\left[Y^{k} \geqslant \frac{c_{k}}{\varepsilon_{k}}\right] \geqslant \alpha .
$$

Это противоречит ограниченности множества $K_{0}^{1}$ в $L_{0}$. Обратно, предположим, что $K_{0}^{1}$ не ограничено в $L_{0}$. Тогда существуют $X^{n} \in \mathscr{X}_{1}$, такие, что

$$
\lim _{n \rightarrow \infty} P\left[X_{1}^{n} \geqslant n\right]=\alpha>0
$$

По определению $\mathscr{X}_{1}$ существует последовательность $\bigcup_{n \geqslant 1} X_{1}^{n} \ni X^{k, n} \stackrel{k \rightarrow \infty}{\longrightarrow}$ $X^{n}$ в топологии Эмери. Поэтому $X_{1}^{k, n} \stackrel{k \rightarrow \infty}{\longrightarrow} X_{1}^{n}$ по вероятности. Для 
каждого $n \in \mathbb{N}$ мы можем выбрать $k_{n}$ так, что

$$
\lim _{n \rightarrow \infty} P\left[X_{1}^{k_{n}, n} \geqslant \frac{n}{2}\right] \geqslant \frac{\alpha}{2} .
$$

Тогда $Y^{n}:=X^{k_{n}, n} / \sqrt{n}$ дает (AА1). Предложение 4.1 доказано.

Предложение 4.2. (NA) $+(\mathrm{NUPBR}) \Leftrightarrow(\mathrm{NA})+(\mathrm{NAA} 1) \Leftrightarrow$ (NAFLVR).

Д о к а з а т е л ь с в о. Доказательство аналогично [8, лемма 2.2]. Для удобства читателя мы приведем соответствующие аргументы в нашей постановке. Сначала покажем, что (NAFLVR) $\Rightarrow(\mathrm{NA})+(\mathrm{NAA} 1)$. Из (4.1) условие (NA) следует тривиально. Предположим существование (AА1). Тогда существует последовательность $X^{k}$, как в определении 4.1. Задавая $f^{k}:=X_{1}^{k} \wedge 1 \in C$ и продолжая, как в [8, лемма $\left.\left.\left.2.2, \mathrm{a}\right) \Rightarrow \mathrm{b}\right)\right]$, получаем противоречие с (NAFLVR).

Обратно, чтобы показать (NA) + (NAA1) $\Rightarrow$ (NAFLVR), предположим, что условие (NAFLVR) не выполняется. Тогда существуют $f^{n} \in C$ и $f \geqslant 0$, такие, что $P[f>0]>0$ и $\left\|f^{n}-f\right\|_{L^{\infty}} \rightarrow 0$. Поэтому существуют $X_{1}^{n} \in \mathscr{X}$, такие, что $X_{1}^{n} \geqslant f_{n} \geqslant-\left\|\left(f^{n}\right)^{-}\right\|_{L^{\infty}}=:-\varepsilon_{n} \rightarrow 0$. По лемме 4.1 условие (NA) влечет, что из $X^{n} \in \varepsilon_{n} \mathscr{X}_{1}$, и $X^{n} / \sqrt{\varepsilon_{n}}$ следует (АА1). Предложение 4.2 доказано.

5. Почему альтернативные множества процессов капитала портфелей не пригодны на больших финансовых рынках. Вместо множества $\mathscr{X}$, введенного в определении 2.2 , мы рассмотрим здесь две другие возможности задания множества процессов капитала портфелей на больших финансовых рынках. В обоих случаях у нас нет желаемого существования эквивалентной разделяющей меры, что, таким образом, иллюстрирует важность взятия замыкания множества 1допустимых портфелей в топологии Эмери, как это сделано в определении 2.2 .

5.1. Важность аппроксимации равномерно допустимыми процессами капитала портфелей. Рассмотрим множество процессов капитала портфелей

$$
\overline{\mathscr{X}}^{\mathbb{S}}=\bar{\bigcup}_{\lambda>0} \bigcup_{n \geqslant 1} \lambda \mathscr{X}_{1}^{n}{ }^{\mathbb{S}} .
$$

В этом случае невозможно заключить, что разделяющая мера для $\mathscr{X}$ будет разделяющей для $\overline{\mathscr{X}}^{\mathbb{S}}$. В самом деле, имеется существенная разница во взятии замыкания в топологии Эмери для 1-допустимых портфелей и взятии объединения по всем $\lambda$, как мы сделали в определении 2.2 , и взятии замыкания в топологии Эмери для $\bigcup_{\lambda>0} \bigcup_{n \geqslant 1} \lambda \mathscr{X}_{1}^{n}$. Действительно, 
пример 2 из работы [3] показывает, что

$$
\bigcup_{\lambda>0} \bigcup_{n \geqslant 1} \lambda \mathscr{X}_{1}^{n}=\overline{\mathscr{X}}^{\mathbb{S}} \supset \mathscr{X}=\bigcup_{\lambda>0} \lambda{\overline{\bigcup_{n \geqslant 1} \mathscr{X}_{1}^{n}}}^{\mathbb{S}} .
$$

В этом примере строятся последовательность мартингалов $\left(S^{n}\right)_{n \in \mathbb{N}}$ и последовательность $\mathbb{R}^{n}$-значных стратегий $\mathbf{H}^{n}$, такие, что для всех $n \in \mathbb{N}$

$$
\left(\mathbf{H}^{n} \bullet \mathbf{S}^{n}\right) \geqslant-n
$$

где $\mathbf{S}^{n}=\left(S^{1}, \ldots, S^{n}\right)$. По лемме Анселя-Стрикера (см., например, [4]) получаем, что $\left(\mathbf{H}^{n} \bullet \mathbf{S}^{n}\right)$ является локальным мартингалом для всех $n \in$ $\mathbb{N}$. Тем не менее, в пределе это неверно. В самом деле, как показано в [3, пример 2], последовательность $\left(\mathbf{H}^{n} \bullet \mathbf{S}^{n}\right)$ сходится в топологии Эмери к возрастающему процессу $A_{t}=t$.

Если мы теперь определим $\mathscr{X}_{1}^{n}$ по последовательности мартингалов $\left(S^{n}\right)_{n \in \mathbb{N}}$ аналогично тому, как в разд. 2.1., $\left(\mathbf{H}^{n} \bullet \mathbf{S}^{n}\right) \in n \mathscr{X}_{1}^{n}$ для каждого $n \in \mathbb{N}$ и поэтому, очевидно, также в $\bigcup_{\lambda>0} \bigcup_{n \geqslant 1} \lambda \mathscr{X}_{1}^{n}$. Поэтому предельный процесс $A_{t}=t$ лежит в $\overline{\mathscr{X}}^{\mathbb{S}}$. Но, определенно, не существует меры $Q \sim P$, такой, что $E_{Q}\left[A_{1}\right]=E_{Q}[1] \leqslant 0$. Множество $\mathscr{X}$, наоборот, удовлетворяет свойству (ESM). В самом деле, в этом случае только равномерно допустимые процессы капитала портфелей на малых рынках берутся в расчет, т.е. только те стратегии, для которых $\left(\mathbf{H}^{n} \bullet \mathbf{S}^{n}\right) \geqslant-\lambda$, для некоторого $\lambda \geqslant 0$ и для всех $n \in \mathbb{N}$. Из леммы Фату следует, что соответствующие пределы Эмери являются супермартингалами, откуда свойство (ESM) (и, значит, также (NAFLVR)) выполняется для $\mathscr{X}$.

В частности, этот пример показывает, что множество $\overline{\mathscr{X}}^{\mathbb{S}}$ в общем случае слишком велико и ведет к асимптотическому арбитражу, который должен быть исключен с самого начала. С другой стороны, множество $\bigcup_{\lambda>0} \bigcup_{n \geqslant 1} \lambda \mathscr{X}_{1}^{n}$ без какого-либо замыкания слишком мало, как будет показано в следующем разделе.

5.2. Нет наивному «отсутствию бесплатного ленча с исчезающим риском». Рассмотрим по аналогии с разд. 3.2 следующее множество процессов портфелей:

$$
\bigcup_{\lambda>0} \bigcup_{n \geqslant 1} \lambda \mathscr{X}_{1}^{n}
$$

и аналогичное понятие (NAFLVR) для этого множества, т.е.

$$
\overline{\mathscr{C}} \cap L_{\geqslant 0}^{\infty}=\{0\}
$$

где $\mathscr{C}$ определено в $(3.2)$ и $\overline{\mathscr{C}}$ обозначает замыкание по норме $L^{\infty}$. Более того, обозначим через $K_{0, n}$ множества значений элементов $\bigcup_{\lambda>0} \lambda \mathscr{X}_{1}^{n}$ в 
конечный момент времени $T=1$, т.е. $K_{0, n}$ отвечает за конечные значения портфелей на малом финансовом рынке $n$. Мы говорим, что выполняется условие $\left(\mathrm{NA}_{\text {small }}\right)$, если каждый малый финансовый рынок $n$ удовлетворяет условию отсутствию арбитража, т.е. если $K_{0, n} \cap L_{\geqslant 0}^{0}=\{0\}$ для всех $n \in \mathbb{N}$. Аналогично предложению 4.2, мы затем получаем следующую характеризацию.

\section{Предложение 5.1. Имеет место:}

$$
\overline{\mathscr{C}} \cap L_{\geqslant 0}^{\infty}=\{0\} \Leftrightarrow(\mathrm{NAA} 1)+\left(\mathrm{NA}_{\text {small }}\right)
$$

Можно построить примеры, для которых условия (NAA1) и $\left(\mathrm{NA}_{\text {small }}\right)$ выполняются, но эквивалентной разделяющей меры для $\bigcup_{\lambda>0} \bigcup_{n \geqslant 1} \lambda \mathscr{X}_{1}^{n}$ не существует. В самом деле, рассмотрим следующую последовательность семимартингалов как модель большого финансового рынка: для $t \in[0,1)$ и $n \in \mathbb{N}$ пусть $S_{t}^{n}=0$ и $S_{1}^{n}$ будут независимыми случайными величинами, принимающими два значения -1 и 1 с вероятностями $P\left[S_{1}^{n}=-1\right]=p_{n}$ и $P\left[S_{1}^{n}=1\right]=1-p_{n}$, где $p_{n}>0$ для всех $n \in \mathbb{N}$, но $\lim _{n \rightarrow \infty} p_{n}=0$. Для каждого $n \in \mathbb{N}$ эквивалентная разделяющая мера $Q$ должна удовлетворять $Q\left[S_{1}^{n}=-1\right]=1 / 2$; однако эта вероятность не стремится к 0, следовательно, мера $Q$ не эквивалентна $P$. Заметим, что условие (NA) в более строгом смысле, определенное в начале разд. 4, не выполняется, так как последовательность инвестиций в $n$-й актив, т.е. $\left(\mathbf{H}^{n} \bullet \mathbf{S}^{n}\right)_{1}=S_{1}^{n}-S_{0}^{n}$ с $\mathbf{H}_{t, i}^{n}=1_{[0,1]}(t) \delta_{i n}$, дает в пределе элемент, который почти наверное равен 1 , и поэтому предоставляет (асимптотическую) возможность арбитража, соответствующую асимптотическому арбитражу второго рода, введенному в [9].

Этот пример показывает, что при $\overline{\mathscr{C}} \cap L_{\geqslant 0}^{\infty}=\{0\}$ имеет место $\overline{\mathscr{C}} \subsetneq$ $\overline{\mathscr{C}}^{*}$. Учитывая предложение 4.2 и рассуждения в разд. 3.2 , ключевым для получения FTAP без использования *-слабых замыканий является усиление условия отсутствия арбитража от условия $\left(\mathrm{NA}_{\text {small }}\right)$ до (NA), определенного в начале разд. 4.

\section{6. О $\sigma$-мартингальных мерах на больших финансовых рын-} ках. Цель этого раздела - показать, что, в отличие от классических малых финансовых рынков, (NAFLVR) не влечет существования $\sigma$-мартингальной меры. В самом деле, следующая модель большого финансового рынка представляет собой контрпример.

Пусть $(\Omega, \mathscr{F}, P)=([0,1], \mathscr{B}([0,1]), \lambda)$, где $\lambda$ обозначает меру Лебега. Для $t \in[0,1)$ и $n \in \mathbb{N}$, пусть $S_{t}^{n}=0$ и определим $S_{1}^{n}$ как

$$
S_{1}^{n}(\omega)= \begin{cases}-\frac{1}{\sqrt{\omega}}, & \omega \in\left[0, \varepsilon_{n}\right), \\ \frac{1}{(1-\omega)^{1 /(n+1)},} & \omega \in\left[\varepsilon_{n}, 1\right],\end{cases}
$$


где $\left(\varepsilon_{n}\right)$ является последовательностью, принимающей значения в $(0,1)$. Мы начнем с леммы, которая показывает существование определенной последовательности $\left(\varepsilon_{n}\right)$, такой, что $E\left[S_{1}^{n}\right]=1$ для всех $n \in \mathbb{N}$ и $\varepsilon_{n} \rightarrow 0$ при $n \rightarrow \infty$.

Лемма 6.1. Пусть $\left(S_{1}^{n}\right)$ задается с помощью (6.1). Тогда мьи можем выбрать $\left(\varepsilon_{n}\right)$ так, ито

$$
E\left[S_{1}^{n}\right]=1 \quad \text { для всех } n \in \mathbb{N}
$$

$u \varepsilon_{n} \rightarrow 0$ npu $n \rightarrow \infty$.

Д о к а з а т е л ь с т в о. Заметим, что $E\left[S_{1}^{n}\right]=1$ эквивалентно соотношению

$$
1+2 \sqrt{\varepsilon_{n}}=\frac{n+1}{n}\left(1-\varepsilon_{n}\right)^{n /(n+1)}
$$

На интервале $[0,1]$ функция $x \mapsto f_{n}(x):=(n+1) / n(1-x)^{n /(n+1)}$ (соответствующая правой части) убывает, тогда как $x \mapsto 1+2 \sqrt{x}$ (соответствующая левой части) возрастает. Поэтому для каждого $n$ найдется некоторое $\varepsilon_{n}$, для которого выполнено (6.2). Более того, по тем же соображениям последовательность $\varepsilon_{n}$ убывает и сходится к 0, так как $\lim _{n \rightarrow \infty} f_{n}(x)=1-x$. Лемма 6.1 доказана.

В дальнейшем мы берем $\left(\varepsilon_{n}\right)$ как в лемме 6.1. Более того, мы рассматриваем фильтрацию, порожденную $\left(S_{t}^{n}\right)_{t \in[0,1], n \in \mathbb{N}}$, так что все предсказуемые стратегии сводятся к детерминированным. Конечное значение портфеля из первых $n$ активов поэтому имеет вид $X_{1}=\sum_{k=1}^{n} c_{k}\left(S_{1}^{k}-\right.$ $\left.S_{0}^{k}\right)=\sum_{k=1}^{n} c_{k} S_{1}^{k}$ с некоторыми константами $c_{k} \in \mathbb{R}$. Следующая лемма предоставляет необходимое условие, гарантирующее, что $X_{1} \geqslant-1$ (эквивалентно, $\left.X \in \mathscr{X}_{1}^{n}\right)$ и показывает, что $P=\lambda$ - разделяющая мера.

Лемма 6.2. Пусть $\left(S_{1}^{n}\right)$ задается $(6.1)$ с $\left(\varepsilon_{n}\right)$, как в лемме 6.1. Для $n \in \mathbb{N}$ предположим, что $X_{1}=\sum_{k=1}^{n} c_{k} S_{1}^{k} \geqslant-1$. Тогда

$$
\sum_{k \in G_{+}} c_{k} \leqslant \sum_{k \in G_{-}}\left|c_{k}\right|
$$

где $G_{+}=\left\{j \mid c_{j}>0\right\}$ u $G_{-}=\left\{j \mid c_{j}<0\right\}$. Более того, $P=\lambda$ является разделяющей мерой для $\mathscr{X}$.

Д о к а з а т е л ь с т в о. Рассматривая первое утверждение, предположим от противного, что

$$
\alpha:=\sum_{k \in G_{+}} c_{k}-\sum_{k \in G_{-}}\left|c_{k}\right|>0 .
$$

Тогда на множестве $\left[0, \varepsilon_{n}\right)$ получим $X_{1}(\omega)=\sum_{k=1}^{n} c_{k} S_{1}^{k}(\omega)=-\alpha / \sqrt{\omega}$, эта величина не ограничена снизу и поэтому влечет (6.3). Учитывая 
лемму 6.1 , получаем, что

$$
\begin{aligned}
E\left[X_{1}\right] & =\sum_{k \in G_{+}} c_{k} E\left[S_{1}^{k}\right]+\sum_{k \in G_{-}} c_{k} E\left[S_{1}^{k}\right] \\
& =\sum_{k \in G_{+}} c_{k}-\sum_{k \in G_{-}}\left|c_{k}\right| \leqslant 0
\end{aligned}
$$

для всех $X \in \mathscr{X}_{1}^{n}$. По лемме Фату это верно также для всех $X \in \mathscr{X}_{1}$ и, в свою очередь, также для $X \in \mathscr{X}$. Лемма 6.2 доказана.

Лемма 6.3. Пусть $\left(S_{1}^{n}\right)$ задается $(6.1)$ с $\left(\varepsilon_{n}\right)$, как в лемме 6.1. Тогда не существует эквивалентной мартингальной мерь, т.е. нет мерь $Q \sim P=\lambda$, такой, что $E_{Q}\left[S_{1}^{n}\right]=0$ для всех $n$.

Д о к а з а т е л ь с т в о. Предположим противное, что существует такая мера $Q \sim \lambda$, что $E_{Q}\left[S_{1}^{n}\right]=0$ для всех $n$. Тогда

$$
\int_{0}^{\varepsilon_{n}} \frac{1}{\sqrt{\omega}} d Q(\omega)=\int_{\varepsilon_{n}}^{1} \frac{1}{(1-\omega)^{1 /(n+1)}} d Q(\omega)
$$

для всех $n$. Так как $Q \sim \lambda$ и $\varepsilon_{n} \rightarrow 0$, то с необходимостью $Q\left(\left[0, \varepsilon_{n}\right)\right) \rightarrow 0$. Более того, так как $(1 / \sqrt{\omega}) 1_{\left[0, \varepsilon_{1}\right)} \in L^{1}(Q)$, по теореме о мажорируемой сходимости мы получаем, что левая часть (6.4) стремится к 0 , когда $n$ стремится к $\infty$. Соответственно, правая часть также стремится к 0 , следовательно, $Q\left(\left[\varepsilon_{n}, 1\right]\right) \rightarrow 0$, так как

$$
\int_{\varepsilon_{n}}^{1} \frac{1}{(1-\omega)^{1 /(n+1)}} d Q(\omega) \geqslant Q\left(\left[\varepsilon_{n}, 1\right]\right) .
$$

Это противоречит эквивалентности $Q$ и $\lambda$ и доказывает утверждение. Лемма 6.3 доказана.

Комбинируя приведенные выше леммы, получаем следующий результат.

Предложение 6.1. Пусть $\left(S_{1}^{n}\right)$ задается (6.1) c $\left(\varepsilon_{n}\right)$, как в лемме 6.1. Тогда существует эквивалентная разделяющая мера (а именно $P=\lambda$ ) но не эквивалентная мартингальная мера.

Д о к а з а т е л с т в о. Доказательство является следствием лемм 6.2 и 6.3 .

3 а м е ч а н и е 6.1. Заметим, что в рассмотренном примере $S^{n}$ будет $\sigma$-мартингалом тогда и только тогда, когда он будет мартингалом. Поэтому мы доказали, что, вообще говоря, (NAFLVR) не влечет существование эквивалентной $\sigma$-мартингальной меры.

7. Доказательство теоремы 3.1. Мы адаптируем стратегию доказательства из [1] к настоящей постановке. Это означает, в частности, 
что первая часть доказательства следует первоначальному доказательству Ф. Делбана и В. Шахермайера [5] для классической ситуации малого рынка. Первый ряд заключений состоит в следующем. Напомним также предложение 4.2, которое требуется, чтобы разделить (NAFLVR) на (NA) и (NUPBR).

(i) Выпуклый конус $C$, определенный в (3.1), замкнут относительно *-слабой топологии тогда и только тогда, когда $C_{0}$ замкнуто по Фату, т.е. $f \in C_{0}$ для любой последовательности $\left(f_{n}\right)$ в $C_{0}$, равномерно ограниченной снизу и сходящейся почти наверное к $f$ (см. начало разд. 3 в [8] и [5, теорема 2.1]). По существу, рассуждение восходят к А. Гротендику.

(ii) Рассмотрим теперь последовательность $-1 \leqslant f_{n} \in C_{0}$, сходящуюся почти наверное к $f$. Тогда мы можем найти $f_{n} \leqslant g_{n}=Y_{1}^{n}$ с $Y^{n} \in \mathscr{X}$.

(iii) Из леммы 4.1 следует, что каждое $Y^{n} \in \mathscr{X}_{1}$.

(iv) Из условия (NUPBR) следует, что существуют форвардновыпуклые комбинации $\widetilde{Y^{n}} \in \operatorname{conv}\left(Y^{n}, Y^{n+1}, \ldots\right)$, такие, что $\widetilde{Y_{1}^{n}} \rightarrow \widetilde{h_{0}} \geqslant f$ почти наверное.

(v) Из этого следует, что множество $\widehat{K}_{0}^{1} \cap\left\{g \in L_{0} \mid g \geqslant f\right\}$, где $\widehat{K}_{0}^{1}$ обозначает замыкание $K_{0}^{1}$ в $L^{0}$, непусто. Поскольку оно также ограничено в силу (NUPBR) и замкнуто, существует максимальный элемент $h_{0}$ (см. начало разд. 3 в [8] или [5, лемма 4.3]). Поскольку $h_{0} \in \widehat{K}_{0}^{1}$, мы можем найти последовательность семимартингалов $X^{n} \in \mathscr{X}_{1}$, такую, что $X_{1}^{n} \rightarrow h_{0}$ почти наверное и $h_{0}$ - это максимальный элемент, больший $f$, с этим свойством.

3 а м е ч а н и е 7.1. Без ограничения общности мы можем взять последовательность семимартингалов в плотном в топологии Эмери подмножестве $\bigcup_{n \geqslant 1} \mathscr{X}_{1}^{n}$ множества $\mathscr{X}_{1}$. В самом деле, из свойств топологии Эмери очевидно, что мы можем найти последовательность в плотном подмножестве, такую, что все еще $X_{1}^{n} \rightarrow h_{0}$ п.н.

(vi) Построенная ранее «максимальная» последовательность семимартингалов $X^{n} \in \bigcup_{n \geqslant 1} \mathscr{X}_{1}^{n}$ сходится равномерно по вероятности, т.е. $\left|X^{n}-X\right|_{1}^{*} \rightarrow 0$ по вероятности, к некоторому càdlàg процессу $X$ (см. [8, лемма 3.2] или [5, лемма 4.5]).

Как и в [1], наша цель теперь состоит в том, чтобы показать, что последовательность $\left(X^{n}\right)$, построенная в п. (vi), которая сходится равномерно к $X$ по вероятности, также сходится к $X$ в топологии Эмери. Из этого будет следовать, что $h_{0}=\lim _{n \rightarrow \infty} X_{1}^{n}=X_{1} \in K_{0}^{1}$, поскольку $\mathscr{X}_{1}$ замкнуто в топологии Эмери. Отсюда, в свою очередь, следует, что $f \in C_{0}$, что завершает доказательство шага (i) выше.

Теорема 7.1. Пусть $\mathscr{X}_{1}={\overline{\bigcup_{n \geqslant 1} \mathscr{X}_{1}^{n}}}^{\mathbb{S}}$ удовлетворяет условию (NUPBR). Пусть $\left(X^{n}\right)_{n \geqslant 0} \in \bigcup_{n \geqslant 1} \mathscr{X}_{1}^{n}-$ последовательность семимартингалов, которая сходится равномерно по вероятности $\kappa X$, 
причем $X_{1}$ - максимальный элемент в $\widehat{K_{0}^{1}}$, где $\widehat{K_{0}^{1}}$ обозначает замькание $K_{0}^{1}$ в $L^{0}$. Тогда $X^{n} \rightarrow X$ в топологии Эмери.

Д о к а з а т е л ь с т в о. В силу предложения A.1, (NUPBR) влечет свойство (P-UT) для $\left(X^{n}\right)$. Поэтому теорема является следствием предложения А.2 и предложения 7.1 ниже. Теорема 7.1 доказана.

Для последовательности семимартингалов $\left(X^{n}\right)_{n \geqslant 0}$ с $X_{0}^{n}=0$ и некоторого $a>0$ рассмотрим следующее разложение

$$
X^{n}=B^{n, a}+M^{n, a}+\check{X}^{n, a},
$$

где $\check{X}^{n, a}=\sum_{s \leqslant t} \Delta X_{s}^{n} 1_{\left\{\left|\Delta X_{s}^{n}\right|>C\right\}}, B^{n, a}$ - предсказуемая компонента конечной вариации и $M^{n, a}$ - локально мартингальная компонента канонического разложения специального семимартингала $X^{n}-\check{X}^{n, C}$.

Предложение 7.1. Пусть $\mathscr{X}_{1}={\overline{\bigcup_{n \geqslant 1} \mathscr{X}_{1}^{n}}}^{\mathbb{S}}$ удовлетворяет условию (NUPBR) и пусть $\left(X^{n}\right)_{n \geqslant 0} \in \bigcup_{n \geqslant 1} \mathscr{X}_{1}^{n}$ - последовательность семимартингалов, которая сходится равномерно по вероятности $\kappa$ $X$, причем $X_{1}$ - максимальный элемент в $\widehat{K_{0}^{1}}$. (Здесь $\widehat{K_{0}^{1}}$ обозначает замькание $K_{0}^{1}$ в $L^{0}$.) Рассмотрим разложения семимартингалов вида (7.1) для $\left(X^{n}\right)$ и $X$ и предположим, ито $M^{n, a} \rightarrow M^{a}$ u $\check{X}^{n, a} \rightarrow \check{X}^{a}$ в топологии Эмери. Тогда $B^{n, a} \rightarrow B^{a}$ в топологии Эмери.

Д о к а з а т е л ь с т в о. Мы адаптируем доказательство предложения 5.5 в [1] с учетом ослабленного свойства конкатенации, как в замечании 2.2. Пусть $\left(X^{n}\right) \in \bigcup_{n \geqslant 1} \mathscr{X}_{1}^{n}$. Тогда $X^{n} \in \mathscr{X}_{1}^{A^{n}}$ для конечных подмножеств $A^{n} \subseteq I$. Определим процессы $Y^{n}:=M^{n, a}+\check{X}^{n, a}$, которые сходятся в топологии Эмери по предположению.

Предположим от противного, что процессы $\left(B^{n, C}\right)$ не сходятся в топологии Эмери. Тогда они не образуют последовательность Коши и существуют такие $i_{k}, j_{k} \rightarrow \infty$, что

$$
P\left[\int_{0}^{1} d\left|B_{s}^{i_{k}, a}-B_{s}^{j_{k}, a}\right|>2 \gamma\right] \geqslant 2 \gamma>0 .
$$

Пусть $B$ - предсказуемый возрастающий процесс, доминирующий все $B^{n, C}$. Положим $r^{i_{k}}:=d B^{i_{k}, a} / d B, r^{j_{k}}:=d B^{j_{k}, a} / d B$ и $\Gamma_{k}:=\left\{r^{i_{k}} \geqslant r^{j_{k}}\right\}$.

Тогда мы можем заключить, предполагая $i_{k} \wedge j_{k} \geqslant i_{k-1} \vee j_{k-1}$ и, возможно, меняя $i_{k}$ и $j_{k}$, что

$$
P\left[\left(\left(r^{i_{k}}-r^{j_{k}}\right) 1_{\Gamma_{k}} \bullet B\right)_{1}>\gamma\right] \geqslant \gamma>0 .
$$

Возьмем $\alpha_{k} \downarrow 0$ и определим $\bar{X}^{k}:=1_{\Gamma_{k}} \bullet X^{i_{k}}+1_{\left(\Gamma_{k}\right)^{c}} \bullet X^{j_{k}}$. Заметим, что по лемме $2.1 \bar{X}^{k} \in \mathscr{X}_{1}^{A^{i}{ }^{\prime} \cup A^{j_{k}}}$. Теперь определим

$$
\sigma_{k}=\inf \left\{t \mid\left(1_{\Gamma_{k}} \bullet Y^{i_{k}}\right)_{t}+\left(1_{\left(\Gamma_{k}\right)^{c}} \bullet Y^{j_{k}}\right)_{t}<Y_{t}^{i_{k}} \vee Y_{t}^{j_{k}}-\alpha_{k}\right\}
$$


Заметим, что $\bar{Y}^{k}-Y^{i_{k}}=1_{\left(\Gamma_{k}\right)^{c}} \bullet\left(Y^{j_{k}}-Y^{i_{k}}\right)$ и $\bar{Y}^{k}-Y^{j_{k}}=1_{\Gamma_{k}} \bullet\left(Y^{i_{k}}-Y^{j_{k}}\right)$ сходятся к 0 в топологии Эмери и, стало быть, также равномерно по вероятности. Мы можем поэтому выбрать достаточно быстро растущие $\left(i_{k}, j_{k}\right)$, чтобы обеспечить $P\left[\sigma_{k}<\infty\right] \rightarrow 0$. Положим теперь $\widetilde{X}^{k}:=1_{\left[0, \sigma_{k}\right]}$ $\bar{X}^{k}$. По лемме 7.1 (см. ниже) $\widetilde{X}^{k} \in\left(1+\alpha_{k}\right) \mathscr{X}_{1}^{A^{i} \cup \cup A^{j_{k}}} \subset\left(1+\alpha_{k}\right) \bigcup_{n \geqslant 1} \mathscr{X}_{1}^{n}$, и мы имеем следующее представление:

$$
\begin{aligned}
\tilde{X}_{1}^{k} & =\left(1_{\Gamma_{k} \cap\left[0, \sigma_{k}\right]} \bullet X^{i_{k}}\right)_{1}+\left(1_{\left(\Gamma_{k}\right)^{c} \cap\left[0, \sigma_{k}\right]} \bullet X^{j_{k}}\right)_{1} \\
& =X_{1 \wedge \sigma_{k}}^{j_{k}}+\left(1_{\Gamma_{k} \cap\left[0, \sigma_{k}\right]} \bullet\left(X^{i_{k}}-X^{j_{k}}\right)\right)_{1} \\
& =X_{1 \wedge \sigma_{k}}^{j_{k}}+\left(1_{\Gamma_{k} \cap\left[0, \sigma_{k}\right]} \bullet\left(Y^{i_{k}}-Y^{j_{k}}\right)\right)_{1}+\xi_{k},
\end{aligned}
$$

где $\xi_{k}=\left(1_{\Gamma_{k} \cap\left[0, \sigma_{k}\right]} \bullet\left(B^{i_{k}, C}-B^{j_{k}, C}\right)\right)_{1}=\left(1_{\Gamma_{k}}\left(r^{i_{k}}-r^{j_{k}}\right) \bullet B\right)_{1 \wedge \sigma_{k}}$. Применяя $[8$, лемма $\mathrm{A}]$ к $\xi_{k}$, получаем, что форвардно-выпуклые комбинации последовательности $\xi_{k}$ сходятся к случайной величине $\eta \geqslant 0$ с $\eta \neq 0$. Обозначая максимальный элемент, к которому сходятся $X_{1}^{j_{k}}$, через $h_{0} \in \widehat{K}_{0}^{1}$, получаем, в силу сходимости $\left(Y^{n}\right)$ в топологии Эмери, что форвардновыпуклые комбинации последовательности $\widetilde{X}_{1}^{k}$ сходятся к $h_{0}+\eta$. Поскольку $\widetilde{X}^{k} \in\left(1+\alpha_{k}\right) \mathscr{X}_{1}$, приходим к противоречию с тем, что $h_{0} \in \widehat{K}_{0}^{1}$ максимально. Предложение 7.1 доказано.

Лемма 7.1. Пусть $X^{k} \in \mathscr{X}_{1}^{A^{k}}, X^{l} \in \mathscr{X}_{1}^{A^{l}}$ (следовательно, $X^{k}, X^{l} \in$ $\mathscr{X}^{A^{k} \cup A^{l}}$ ) и $\alpha>0$. Рассмотрим разложение (7.1) для $X^{k} u X^{l}$, m.е.

$$
X^{i}=B^{i, C}+M^{i, C}+\check{X}^{i, C}=: B^{i, C}+Y^{i}, \quad i=k, l .
$$

Пусть $B$ - предсказуемый возрастающий прочесс, доминирующий $B^{i, C}$. Положим $r^{i}=d B^{i, C} / d B$ для $i=k, l u$

$$
\sigma:=\inf \left\{t \mid\left(1_{\Gamma} \bullet Y^{k}\right)_{t}+\left(1_{\Gamma^{c}} \bullet Y^{l}\right)_{t}<Y_{t}^{k} \vee Y_{t}^{l}-\alpha\right\},
$$

где $\Gamma=\left\{r^{k} \geqslant r^{l}\right\}$. Тогда

$\tilde{X}=1_{\Gamma \cap[0, \sigma]} \bullet X^{k}+1_{\Gamma^{c} \cap[0, \sigma]} \bullet X^{l} \in(1+\alpha) \mathscr{X}_{1}^{A^{k} \cup A^{l}} \subseteq(1+\alpha) \bigcup_{n \geqslant 1} \mathscr{X}_{1}^{n} \subseteq(1+\alpha) \mathscr{X}_{1}$.

Д о к а з а т е л ь с т в о. Заметим, что $1_{\Gamma} \bullet B^{k, C}+1_{\Gamma^{c}} \bullet B^{l, C} \geqslant B^{k, C} \vee$ $B^{l, C}$. Таким образом, на $[0, \sigma]$

$\tilde{X} \geqslant B^{k, C} \vee B^{l, C}+Y^{k} \vee Y^{l}-\alpha \geqslant\left(B^{k, C}+Y^{k}\right) \vee\left(B^{l, C}+Y^{l}\right)-\alpha=X^{k} \vee X^{l}-\alpha$.

В момент $\sigma$ скачок $\Delta \widetilde{X}$ удовлетворяет $\Delta \widetilde{X}_{\sigma}=1_{\Gamma} \Delta X_{\sigma}^{k}+1_{\Gamma^{c}} \Delta X_{\sigma}^{l}$ и, следовательно, $\widetilde{X}_{\sigma} \geqslant-1-\alpha$, потому что левый предел не меньше, чем $X^{k} \vee X^{l}-\alpha$. Лемма 7.1 доказана. 


\section{Приложение А. Некоторые технические результаты.}

$\mathrm{O}$ п р е д е л е н и е A.1. Положительный càdlàg согласованный процесс $D$ называется супермартингальным дефлятором для $1+\mathscr{X}_{1}$, если $D_{0} \leqslant 1$ и $D(1+X)$ есть супермартингал для всех $X \in \mathscr{X}_{1}$.

Теорема A.1. Пусть $\mathscr{X}_{1}$ - множество семимартингалов, удовлетворяющее определению 2.2 и условию (NUPBR). Тогда существует супермартингальный дефлятор $D$ для $1+\mathscr{X}_{1}$.

Д о к а з а т е л ь с т в о. Как в доказательстве теоремы 4.5 в [1], утверждение теоремы следует из [12, лемма 2.3]. В самом деле, следуя доказательству теоремы 4.5 в [1] и используя вариант свойства конкатенации из леммы 2.1 , легко видеть, что для $\bigcup_{n \geqslant 1} \mathscr{X}_{1}^{n}$ имеет место свойство разветвленной выпуклости (fork-convexity). Поэтому существует супермартингальный дефлятор $D$ для $\bigcup_{n \geqslant 1} \mathscr{X}_{1}^{n}$. По лемме Фату $D$ будет также супермартингальным дефлятором для замыкания $\mathscr{X}_{1}$ в топологии Эмери. Теорема А.1 доказана.

Следующее предложение соответствует [1, предложение 4.10], и его доказательство полностью аналогично в настоящей постановке.

Предложение A.1. Пусть $\mathscr{X}_{1}$ удовлетворяет условию (NUPBR), и пусть $\left(X^{n}\right)_{n \geqslant 0} \in \mathscr{X}_{1}$ - любая последовательность семимартингалов. Тогда $\left(X^{n}\right)$ удовлетворяет свойству (P-UT).

Следующее предложение - это переформулировка $[18$, предложение 1.10] и соответствует [1, предложение 5.2].

Предложение А.2. Пусть $\left(X^{n}\right)_{n \geqslant 0}-$ последовательность семимартингалов с $X_{0}^{n}=0$, которая сходится равномерно по вероятности $\kappa X$. Предположим, кроме того, что для этой последовательности выполнено свойство (P-UT), и рассмотрим разложения вида (7.1) для $\left(X^{n}\right)$ и $X$. Тогда существует некоторое $C>0$, такое, ито $M^{n, a} \rightarrow M^{a}$ и $\check{X}^{n, a} \rightarrow \check{X}^{a}$ в топологии Эмери, и $B^{n, C} \rightarrow B^{C}$ равномерно по вероятности.

\section{СПИСОК ЛИТЕРАТУРЫ}

1. Cuchiero C., Teichmann J. A convergence result in the Emery topology and a variant of the proof of the fundamental theorem of asset pricing. Preprint, 2014, arXiv:1406.4301.

2. De Donno M., Guasoni P., Pratelli M. Super-replication and utility maximization in large financial markets. — Stochastic Process. Appl., 2005, v. 115, № 12, p. 2006-2022.

3. De Donno M., Pratelli $M$. Stochastic integration with respect to a sequence of semimartingales. - Lecture Notes in Math., 2006, v. 1874, p. 119-135.

4. De Donno M., Pratelli M. On a lemma by Ansel and Stricker. - Lecture Notes in Math., 2007, v. 1899, p. 411-414.

5. Delbaen $F$., Schachermayer $W$. A general version of the fundamental theorem of asset pricing. - Math. Ann., 1994, v. 300, № 3, p. 463-520. 
6. Emery M. Une topologie sur l'espace des semimartingales. - Lecture Notes in Math., 1979. v. 721 , p. $260-280$.

7. Ingersoll J. Theory of Financial Decision Making. Lanham: Rowman \& Littlefield Publishers, 1987, 474 p.

8. Kabanov Y.M. On the FTAP of Kreps-Delbaen-Schachermayer. In Statistics and Control of Stochastic Processes (Moscow, 1995/1996). River Edge, NJ: World Scientific Publishers, 1997, p. 191-203.

9. Кабанов Ю.М., Крамков Д. О. Большие финансовые рынки: асимптотический арбитраж и контигуальность. - Теория вероятн. и примен., 1994, т. 39, в. 1, c. $222-229$.

10. Kabanov Y.M., Kramkov D. O. Asymptotic arbitrage in large financial markets. Finance Stoch., 1998, v. 2, № 2, p. 143-172.

11. Karatzas I., Kardaras C. The numéraire portfolio in semimartingale financial models. - Finance Stoch., 2007, v. 11, № 4, p. 447-493.

12. Kardaras $C$. On the closure in the Emery topology of semimartingale wealth-process sets. - Ann. Appl. Probab., 2013, v. 23, № 4, p. 1355-1376.

13. Klein I. A fundamental theorem of asset pricing for large financial markets. - Math. Finance, 2000, v. 10, № 4, p. 443-458.

14. Klein I., Schachermayer W. Asymptotic arbitrage in non-complete large financial markets. - Теория вероятн. и примен., 1996, т. 41, в. 4, с. 927-934.

15. Klein I., Schmidt T., Teichmann J. When roll-overs do not qualify as numéraire: bond markets beyond short rate paradigms, preprint http://arxiv.org/abs/1310.0032, 2013.

16. Kreps D.M. Arbitrage and equilibrium in economies with infinitely many commodities. - J. Math. Econom., 1981, v. 8, № 1, p. 15-35.

17. Mémin J. Espaces de semi martingales et changements de probabilité. - Z Wahrscheinlichkeitstheor. verw. Geb., 1980, v. 52, № 1, p. 9-39.

18. Mémin J., Stominski L. Condition UT et stabilité en loi des solutions d'équations différentielles stochastiques. - Séminaire de probabilités de Strasbourg, 1991, v. 25, p. $162-177$.

19. Schaefer H. H., Wolff M.P. Topological vector spaces. New York: Springer-Verlag, 1999, $346 \mathrm{p}$.

20. Yan J. A. Caractérisation d'une classe d'ensembles convexes de $L^{1}$ ou $H^{1}$. - Lecture Notes in Math., 1980, v. 784, p. 220-222. 